\title{
Effects of different circuit training protocols on body mass, fat mass and blood parameters in overweight adults
}

\author{
Valentina Contrò, ${ }^{1}$ Antonino Bianco, ${ }^{2}$ Jill Cooper, ${ }^{2}$ Alessia Sacco, ${ }^{2}$ Alessandra Macchiarella, ${ }^{2}$ \\ Marcello Traina, ${ }^{2}$ Patrizia Proia ${ }^{2}$ \\ ${ }^{1}$ Department of Economical and Statistical Sciences, University of Palermo; ${ }^{2}$ Department of Psychological, \\ Pedagogical and Educational Sciences, University of Palermo, Palermo, Italy
}

\begin{abstract}
Benefits of exercise are known for a long time, but mechanisms underlying the exercise mode recommendations for specific chronic cardiovascular diseases remain unclear. The aim of this study was to compare the effects of different circuit training protocols in order to determine which is the best for weight loss and for specific overweight-related disorders. Forty-five female sedentary overweight participants from 20 to 50 years (average $31.8 \pm 11.2$ ) were enrolled and assigned to three different groups; each group was compared with a control normal-weight group. Three different circuit protocols were randomly assigned to each overweight group: aerobictone-aerobic (ATA), aerobic-circuit-aerobic (ACA) and mini-trampoline circuit (MTC), while control group performed a classic circuit weight training (CWT). Every group trained three times per week, for 12 weeks. The results show that ATA group reduced body fat and total body mass more than other groups $(\mathrm{P}<0.001 ; \mathrm{P}=0.007)$. ACA group reduced total body mass in significant statistical way $(\mathrm{P}=0.032)$, as well as body fat $(\mathrm{P}<0.001)$ and low-density lipoprotein cholesterol $(\mathrm{P}=0.013)$. In MTC group there was a significant reduction in every parameter we analyzed (total body mass, body fat and lipid profile: $\mathrm{P}<0.001)$. CWT group has shown a significant loss
\end{abstract}

Correspondence: Patrizia Proia, Department of Psychological, Pedagogical and Educational Sciences, University of Palermo, via Pascoli 6, 90144 Palermo, Italy.

Tel/Fax: +39.091.23899906.

E-mail: patrizia.proia@unipa.it

Acknowledgements: we would like to show our gratitude to all the participants of the study. We thank Dr. Jill Cooper for sharing her pearls of wisdom during the course of this research.

Key words: Weight loss; Overweight; Circuit training; Lipid profile.

Received for publication: 8 September 2016.

Revision received: 3 Janaury 2017.

Accepted for publication: 19 Janaury 2017.

(C) Copyright V. Contrò et al., 2017

Licensee PAGEPress, Italy

Journal of Biological Research 2017; 90:6279

doi:10.4081/jbr.2017.6279

This article is distributed under the terms of the Creative Commons Attribution Noncommercial License (by-nc 4.0) which permits any noncommercial use, distribution, and reproduction in any medium, provided the original author(s) and source are credited. only in body fat $(\mathrm{P}<0.001)$. Every circuit protocol is optimal for reducing body fat and total body mass: however, MTC protocol has shown the best results on lipid profile.

\section{Introduction}

Many of the biochemical and physiological adaptations that follow physical activity occur in response to an increased energy demand in muscle cells that can be modifies with intensity, duration of work and rest intervals.

The aforesaid adaptations, which are both cellular and systemic, are specific to the type of training performed, for example, endurance or strength; though, the reasons behind the recommendations on how training programs can be used for specific health benefits remain unclear.

In the past, many guidelines have focused on resistance training or aerobic training to facilitate weight loss or weight maintenance, but recently it has been suggested that resistance training can also be effective for reducing fat mass, ${ }^{1,2}$ although the literature in this regard is still rather hazy.

Some researchers have suggested that a circuit training composed of endurance and resistance training it may be preferred to one focused only on a single mode of exercise. ${ }^{3-5}$

Both resistance training and aerobic training are prescribed for sedentary and obese subject, because they promote an improvement of blood pressure and heart rate, body composition, biochemical markers (insulin, glucose, cholesterol, etc.), as well as strenght and aerobic capacity. ${ }^{6-8}$

A type of combinated-training is the circuit training (CT), which includes both multi-joint resistance training and aerobic exercise. The proportion of the two types of training is established and bodyweight exercises or with small tools keep high heart rate. The training session includes several stations where different exercises are performed for a set time; after the appointed time the station is changed as quickly as possible without rest period.

As shown by Paoli and collegues, the effect of CT on various physiological measurements is intensity-dependent: subjects who performed a high intensity circuit training for 12 weeks had the greatest reductions in body weight, percentage of body fat, waist circumference, and blood lactate during a submaximal exercise and a greater improvement than the low intensity circuit group. ${ }^{9}$

In the present study we compared four different CT protocols and we evaluated the effects on several key physiological health markers (like body fat mass and dyslipidemia) in overweight subjects with the purpose of establishing which protocol is the best.

The estimated number of people with dyslipidemia is expected 
to reach 78 million in the most important countries by $2022 .{ }^{10}$

Hyperlipidemia is a leading cause of morbidity and mortality worldwide and one of the major and modifiable risk factors for cardiovascular disease investigated in this study. ${ }^{11}$

\section{Materials and Methods}

Forty-five female sedentary (exercising $<1-2$ times/wk) overweight [body mass index $(\mathrm{BMI})>25$ ] from 20 to 50 (mean $31.8 \pm 11.2$ ) years old, who could have mild to moderate dyslipidemia (either total cholesterol $>220 \mathrm{mg} / \mathrm{dL}$, low-density lipoprotein (LDL) cholesterol 130-190 mg/dL, HDL cholesterol<40 md/dL), were enrolled in the study and were assigned to three different groups (15 subjects each); each group was compared with a control normal-weight group of 15 subjects $(\mathrm{BMI}<25)$.

The overweight groups were randomly assigned to one of three different circuit protocols: aerobic-tone-aerobic (ATA), aerobiccircuit-Aerobic (ACA) and mini-trampoline circuit (MTC), while control group performed a classical circuit weight training (CWT).

\section{Training protocols}

The participants trained 3 times a week (with at least 1 day of rest between session) and 60 minutes per session for 12 weeks under the supervision of qualified sport scientists and researchers.

To set up the intensity of aerobic training we have used the maximum heart rate (HR) obtained during the maximal graded test. Each session included a warm-up and cool-down period involving $5 \mathrm{~min}$ of low-intensity and light stretching activities.

Each protocol corresponds to a training performed in circuit, that is doing multiple sets of exercises using medium to high repetitions, low intensity, without breaks, or with very short breaks between exercises. The exercises with weights can be done using free weights, strength machines, and calisthenics, and their execution is performed without any pause.

The advantages of circuit training were mainly due to a stimulus on the improvement of strength, hypertrophy, muscular endurance and aerobic capacity in a single training session, able to generate the anaerobic and aerobic workout benefits, although in a generally lower way when compared to conventional methods.

CWT it is a variation of the resistance training, performed in circuit. Intensity was maintained at $60 \%$ of HR max. Partecipants performed 10 exercises for the main muscle groups, 2 sets of 15 reps.

ATA is a training method in which the aerobic (cardio) part is mixed with the anaerobic (resistance training) part in the same session, with the aim to emphasize weight loss compared to traditional aerobic methods. Participants trained by alternating $10^{\prime}$ of endurance on treadmill at $70 \%$ of HR max with training at 5-resistance exercise stations (push ups; lateral shoulder raise; abdominal crunches; squats; calf).

ACA mixes the typical weight training exercises with the traditional aerobic cardiovascular workout, with the aim of producing both improvements in terms of strength and muscle mass, and on cardiovascular capacity. In this kind of protocol, the weight training exercises are alternated with 5 aerobic stations, called active recovery (jumping rope, jumping jack, skip, mountain climbers, jog on the spot); the duration of which varies from 30-60 seconds to 2-3 minutes or more, with moderate intensity.

MTC mixes aerobic and anaerobic exercises performed all on a mini trampoline $(122 \mathrm{~cm}$ diameter modified mini trampoline designed by Jill Cooper). The exercise sequences stimulate alternately upper and lower muscle groups: this technique is designed to stimulate the cardiovascular engagement follows the redistribution of blood flow in muscle groups far from each other.

\section{Experimental procedures}

Weight was measured to the nearest $0.01 \mathrm{~kg}$ using an electronic scale (BWB-800 Medical Scales; Tanita Corporation, Arlington Heights, IL, USA), and height to the nearest $0.01 \mathrm{~m}$ using a Harpenden portable stadiometer (Holtain Ltd, Crosswell, UK).

Body composition was assessed using bioelectrical impedance analysis (BIA; Akern Bioresearch, Pontassieve, Italy), which is a non-invasive and portable method for the estimation of fluid compartments, fat, and fat-free mass.

The maximal graded tests were performed on an electronically braked cycle ergometer (type Monark), under the supervision of a cardiologist, using a Polar heart rate monitor (Polar, Kemple, Finland).

Venous blood samples were taken at rest, before starting the fitness training programs and repeated at the end of them (after 12 weeks).

Blood plasma was obtained through blood centrifugation (1077 g x 5') and it was used to determine lipid profile: total cholesterol, high-density lipoprotein cholesterol (HDL) and triglycerides (TG) were measured by an enzymatic colorimetric method using a Modular D2400 (Hoffman-La Roche Ltd, Basel, Switzerland). LDL-C fraction was calculated from Friedewald's formula: LDL-C=TC - HDL-C - (TG/5).

\section{Results}

In every group we analyzed total body mass, body fat and lipid profile and the results show that the ATA group reduced body fat and and total body mass more than other groups $(\mathrm{P}<0.001$ and 0.007 , respectively) (Table 1 ). The ACA reduced total body mass in significant statistical way $(\mathrm{P}=0.032)$, as well as body fat $(\mathrm{P}<0.001)$ and LDL cholesterol $(\mathrm{P}=0.013)$. Concerning the MTC group, there was a significant reduction in every parameter we analyzed (total body mass, body fat and lipid profile: $\mathrm{P}<0.001$ ). The CG (performed CWT protocol) has shown a significant loss only in body fat $(\mathrm{P}<0.001)$. In conclusion, it appears that all the circuit protocols are optimal exercises for reducing body fat and total body mass: however, MTC protocol has shown the best results on lipid profile.

\section{Statistical analysis}

Multivariate analysis of variance (MANOVA) on difference measures was used to compare weight, BMI, and lipid profiles across all groups. Whenever significant differences in values occurred, multiple comparisons test (useful for determining where significant differences occur between pairs of groups) was performed using a post-hoc Tukey-Kramer test, considered the most powerful method for all pairwise comparisons. Alpha significance level was set at 5\% (and was adjusted for multiple comparisons). Statistica Software, ver. 8.0 was used for the analysis.

\section{Discussion}

Our study compared four different circuit training protocols, trying to determine which can give better results in different situa- 
Table 1. Variables analyzed before and after three different circuit protocols and the control.

\begin{tabular}{|c|c|c|c|c|c|c|c|c|c|c|c|c|}
\hline Variables & & ATA & & & ACA & & & CWT & & & MTC & \\
\hline & Before & After & P & Before & After & $P$ & Before & After & P & Before & After & P \\
\hline MI $\left(\mathrm{kg} / \mathrm{m}^{2}\right)$ & & $25.89 \pm 2.7$ & 0.053 & $26.64 \pm 5.59$ & $26 \pm 5.1$ & $0.002^{*}$ & $25.08 \pm 4.25$ & $24.92 \pm 3.9$ & 0.43 & & $25.02 \pm 3.9$ & $0.000^{*}$ \\
\hline W (Kg) & $67.26 \pm 6.7$ & $65.03 \pm 7.3$ & $0.007^{*}$ & $72.81 \pm 17.0$ & $71.22 \pm 15.8$ & 0.032 & $70.90 \pm 16.6$ & $69.97 \pm 15.6$ & 0.087 & $70.90 \pm 11.0$ & $67.45 \pm 10.2$ & $0.000 *$ \\
\hline $\mathrm{FM}(\mathrm{Kg})$ & $24.24 \pm 5.1$ & $21.40 \pm 5.9$ & $0.000^{*}$ & $23.46 \pm 12.8$ & $21.10 \pm 11.5$ & $0.000^{*}$ & & $17.16 \pm 5.2$ & $0.001^{*}$ & & $19.16 \pm 10.3$ & $0.000 *$ \\
\hline LM (Kg) & & $28.81 \pm 2.05$ & 0.504 & & & & & & & & $52.00 \pm 6.9$ & $0.000^{*}$ \\
\hline $\mathrm{CHOL}(\mathrm{mg} / \mathrm{dL})$ & $168.14 \pm 32.2$ & $169.28 \pm 29.7$ & 0.865 & 171.4 & $162.21 \pm 34.5$ & & & $171.33 \pm 28.3$ & 45 & 228.6 & $191.15 \pm 19.5$ & $0.000^{*}$ \\
\hline TG (mg/dL) & $94.14 \pm 45.9$ & $75.71 \pm 30.3$ & 0.182 & $66.07 \pm 20.4$ & $78.43 \pm 32.7$ & 0.123 & & $83.50 \pm 39.2$ & 0.127 & $191.40 \pm 63.4$ & $149.00 \pm 38.3$ & $0.000^{*}$ \\
\hline HDL (mg/dL) & $48.86 \pm 6.5$ & $48.14 \pm 7.2$ & 0.844 & $51.36 \pm 10.7$ & $51.86 \pm 12.3$ & 0.8 & & $54.67 \pm 15.8$ & 0.095 & $51.35 \pm 14.2$ & $59.30 \pm 12.6$ & $0.000^{*}$ \\
\hline LDL (mg/dL) & $100.28 \pm 24.9$ & $106.15 \pm 28.1$ & 0.194 & $107.64 \pm 36.2$ & $94.78 \pm 30.7$ & 0.013 & $101.42 \pm 25.6$ & $100.42 \pm 26.9$ & 0.842 & $139.00 \pm 23.2$ & $99.86 \pm 19.7$ & $0.000^{*}$ \\
\hline $\mathrm{GL}(\mathrm{mg} / \mathrm{dL})$ & $91.71 \pm 9.8$ & $95.14 \pm 5.9$ & 0.291 & $87.64 \pm 10.3$ & $85.07 \pm 9.1$ & 0.25 & $90.0 \pm 7.3$ & $90.5 \pm 9.2$ & 0.75 & $130.5 \pm 24.2$ & $106.2 \pm 18.4$ & $0.000^{*}$ \\
\hline
\end{tabular}

ATA, aerobic-tone-aerobic; ACA, aerobic-circuit-aerobic; CWT, circuit weight training; MTC, mini-trampoline circuit; BMI, body mass index; W, weight; BFM, body fat mass; BLM, body lean mass; CHOL, total cholesterol; TG, triglycerides; HDL, high density lipoprotein cholesterol; LDL, low-density lipoprotein; GL, glucose. The significance levels of the differences observed between analyzed time-points were assigned using Ttest for Dependent samples (Statistica Software). *Differences statistically significant.

tions of increased cardiovascular risk and we can assert all of them, with or without weights, with no breaks and low to moderate intensity, repeated 3 times a week for 12 weeks had a high impact on examined health markers.

One of the main positive factors of a CT is the variety of exercises, which have a quite short duration and are frequently changed, that might be more appealing to a sedentary subject who considers boring to workout.

Literature data are numerous, specifically those concerning the female sex, ${ }^{12}$ which highlights the positive effects of both aerobic training and strength training, in all ages and in particular conditions such as premenopausal.

Concerning timing of intervention (12 weeks), in literature were confirmed remarkable results, for kind of protocols that compared aerobic workouts with anaerobic workouts, both for training with moderate and progressive intensity. ${ }^{13}$ It supports the assumption that 12-week-long exercise programme may be sufficient for achieving beneficial effect as well as that this effect varies depending on the somatic built of the subject.

\section{Conclusions}

In our study, we noticed that minor effect changes occurred in normal weight group; this can possibly be explained by the fact that it has been administered a medium intensity protocol, and it has recently been shown that the best results, especially in nonobese subjects, are obtained with high intensity circuit protocols. ${ }^{14}$

Moreover, the only parameter that decreases in a statistically significant way, that is body fat mass, oddly does not seem to go in parallel with a statistically significant reduction in cholesterol and triglycerides.

\section{References}

1. Hollowell RP, Willis LH, Slentz CA, et al. Effects of exercise training amount on physical activity energy expenditure. Med Sci Sports Exerc 2004;41:1640-4.

2. Donnelly JB, Jakicic JM, Manore MM, et al. Appropriate physical activity intervention strategies for weight loss and prevention of weight regain for adults. Med Sci Sports Exerc 2009;41:459-71.
3. Bateman LA, Slentz CA, Willis LH, et al. Comparison of aerobic versus resistance exercise training effects on metabolic syndrome. Am J Cardiol 2011;108:838-44.

4. Slentz CA, Bateman LA, Willis LH, et al. Effects of aerobic vs. resistance training on visceral and liver fat stores, liver enzymes, and insulin resistance by HOMA in overweight adults (from STRRIDE AT/RT). Am J Physiol Endocrinol Metab 2011;301:1033-9.

5. Willis LH, Slentz CA, Bateman LA, et al. Effects of aerobic and/or resistance training on body mass and fat mass in overweight or obese adults. J Appl Physiol 2012;113:1831-7.

6. Regaieg S, Charfi N, Abid M. The effects of an exercise training program on body composition and aerobic capacity parameters in Tunisian obese children. Indian $\mathrm{J}$ Endocrinol Metab 2013;17:1040-5.

7. Kostrzewa-Nowak D, Nowak R, Jastrzębski Z, et al. Effect of 12week-long aerobic training programme on body composition, aerobic capacity, complete blood count and blood lipid profile among young woman. Biochem Med (Zagreb) 2015;25:103-13.

8. Miller MB, Pearcey GE, Cahill F, et al. The effect of a shortterm high-intensity circuit training program on work capacity, body composition, and blood profiles in sedentary obese men: a pilot study. Biomed Res Int 2014; 2014:191797.

9. Paoli A, Pacelli QF, Moro T, et al. Effects of high-intensity circuit training, low-intensity circuit training and endurance training on blood pressure and lipoproteins in middle-aged overweight men. Lipids Health Dis 2013;12:131.

10. Ni WQ, Liu XL, Zhuo ZP, et al. Serum lipids and associated factors of dyslipidemia in the adult population in Shenzhen. Lipids Health Dis 2015;14:71.

11. Tannock L, Bhat A. Risk assessment and guidelines for the management of high triglycerides. In: De Groot LJ, BeckPeccoz P, Chrousos G, et al, eds. Endotext. South Dartmouth (MA): MDText.com, Inc.; 2000-2015.

12. Schmitz KH, Hannan PJ, Stovitz SD, et al. Strength training and adiposity in premenopausal women: strong, health, and empowered study. Am J Clin Nutr 2007;86:566-72.

13. Sanal E, Ardic F, Kirac S. Effects of aerobic or combined aerobic resistance exercise on body composition in overweight and obese adults: gender differences. A randomized intervention study. Eur J Phys Rehabil Med 2013;49:1-11.

14. Schmidt W, Anderson K, Graff M, Strutz V. The effect of highintensity circuit training on physical fitness. J Sports Med Phys Fitness 2016:56:534-40. 